Author affiliations and support information (if applicable) appear at the end of this article.

Published at jco.org on June 27, 2017. Clinical trial information: NCT00000611. Corresponding author: Rowan $\mathrm{T}$. Chlebowski, MD, PhD, City of Hope National Medical Center, 1500 E Duarte Rd, Duarte, CA 91010; e-mail: rowanchlebowski@gmail.com.

(c) 2017 by American Society of Clinical Oncology

0732-183X/17/3525w-2919w/\$20.00

\title{
Low-Fat Dietary Pattern and Breast Cancer Mortality in the Women's Health Initiative Randomized Controlled Trial
}

Rowan T. Chlebowski, Aaron K. Aragaki, Garnet L. Anderson, Cynthia A. Thomson, JoAnn E. Manson, Michael S. Simon, Barbara V. Howard, Thomas E. Rohan, Linda Snetselar, Dorothy Lane, Wendy Barrington, Mara Z. Vitolins, Catherine Womack, Lihong Qi, Lifang Hou, Fridtjof Thomas, and Ross L. Prentice

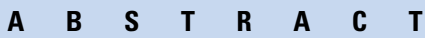

\section{Purpose}

Earlier Women's Health Initiative Dietary Modification trial findings suggested that a low-fat eating pattern may reduce breast cancers with greater mortality. Therefore, as a primary outcome-related analysis from a randomized prevention trial, we examined the long-term influence of this intervention on deaths as a result of and after breast cancer during 8.5 years (median) of dietary intervention and cumulatively for all breast cancers diagnosed during 16.1 years (median) of follow-up.

\section{Patients and Methods}

The trial randomly assigned 48,835 postmenopausal women with normal mammograms and without prior breast cancer from 1993 to 1998 at 40 US clinical centers to a dietary intervention with goals of a reduction of fat intake to $20 \%$ of energy and an increased intake of fruits, vegetables, and grains $(40 \% ; n=19,541)$ or to a usual diet comparison $(60 \% ; n=29,294)$.

\section{Results}

In the dietary group, fat intake and body weight decreased (all $P<.001$ ). During the 8.5-year dietary intervention, with 1,764 incident breast cancers, fewer deaths occurred as a result of breast cancer in the dietary group, which was not statistically significant (27 deaths $[0.016 \%$ per year] $v 61$ deaths [0.024\% per year]; hazard ratio [HR], 0.67; 95\% Cl, 0.43 to $1.06 ; P=.08$ ). During the same period, deaths after breast cancer $(n=134)$ were significantly reduced $(40$ deaths $[0.025 \%$ per year] $v 94$ deaths $[0.038 \%$ per year]; $\mathrm{HR}, 0.65 ; 95 \% \mathrm{Cl}, 0.45$ to 0.94 ; $P=.02$ ) by the dietary intervention. During the 16.1-year follow-up, with 3,030 incident breast cancers, deaths after breast cancer also were significantly reduced (234 deaths $0.085 \%$ per year] $v 443$ deaths $[0.11 \%$ per year]; $\mathrm{HR}, 0.82 ; 95 \% \mathrm{Cl}, 0.70$ to $0.96 ; P=.01$ ) in the dietary group.

\section{Conclusion}

Compared with a usual diet comparison group, a low-fat dietary pattern led to a lower incidence of deaths after breast cancer.

\section{J Clin Oncol 35:2919-2926. (C) 2017 by American Society of Clinical Oncology}

\section{INTRODUCTION}

In the Women's Health Initiative Dietary Modification (WHI DM) trial, 48,835 postmenopausal women were randomly assigned to a dietary modification group or usual diet comparison group to assess low-fat dietary pattern effects on breast cancer incidence. As previously reported, the dietary modification program reduced fat intake; increased fruit, vegetable, and grain intake; and was associated with modest weight loss (3\% loss after 1 year; $-2.2 \mathrm{~kg} ; P<.001) .{ }^{1}$ Dietary intake and body weight differences between randomly assigned groups were maintained throughout the dietary intervention period. ${ }^{1,2}$

At the protocol-specified end of the dietary intervention, after 8.5 years (median) of followup, although the breast cancer incidence rate was lower in the dietary group, the difference was not statistically significant (hazard ratio [HR], 0.92; 95\% CI, 0.84 to $1.01 ; P=.09)$. However, a significant reduction in estrogen receptor-positive $(\mathrm{ER}+)$, progesterone receptor-negative $(\mathrm{PR}-)$ cancers was observed (HR, 0.64; 95\% CI, 0.49 to $0.84 ; P=.04) .^{1}$ No suggestion of reduced breast cancer incidence existed during the limited 
postintervention follow-up, but the significant reduction in ER+, PR-cancers continued (HR, $0.70 ; 95 \%$ CI, 0.56 to 0.88 ). ${ }^{3}$ Although not statistically significant, during the dietary intervention, fewer deaths as a result of breast cancer were observed in the dietary group (HR, $0.77 ; 95 \% \mathrm{CI}, 0.48$ to 1.22 ). ${ }^{1}$

Taken together, these findings suggest that dietary modification reduces breast cancers associated with a greater risk of death, and this possibility has now been examined through 16.1 years (median) of cumulative follow-up. We present results of dietary modification influence on deaths as a result of breast cancer (breast cancer followed by death attributed to the cancer) and on the more general outcome of deaths after breast cancer (breast cancer followed by death as a result of any cause). These end points are examined first through the end of the 8.5-year (median) dietary intervention period and then cumulatively for all breast cancers diagnosed at any time throughout the 16.1-year (median) followup through 2013.

\section{PATIENTS AND METHODS}

\section{Participants}

Design details of the WHI DM trial, which was conducted at 40 US clinical centers with enrollment from 1993 through 1998, have been described previously. ${ }^{4,5}$ Eligible participants were postmenopausal women between 50 and 79 years of age with no previous breast cancer, a dietary fat intake $>32 \%$ of total energy by food frequency questionnaire (FFQ), ${ }^{6}$ and a mammogram not suspicious for cancer. The trial was approved by institutional review boards of the participating clinical centers, and participants provided written informed consent.

\section{Random Assignment}

Participants were randomly assigned to a low-fat dietary pattern intervention group or a usual diet comparison group in a two-to-three ratio for cost efficiency by using a permuted block algorithm stratified by clinical center and age decade, with allocations developed by the WHI Clinical Coordinating Center.

\section{Procedure}

Baseline characteristics were gathered by interview for medication use and by questionnaires for other variables. Body weight and height were measured at baseline and annually, with body mass index calculated as kilograms per meter squared. Physical activity was assessed by questionnaire at baseline, year 3, and year 6, with metabolic equivalent tasks (METs) calculated in MET-hours per week. Mammography screening was performed every 2 years or annually for the $16 \%$ who also participated in a WHI hormone therapy trial. Physicians outside the WHI directed breast cancer therapy.

The low-fat dietary program was designed to reduce fat intake to $20 \%$ of total energy and to increase vegetable, fruit, and grain intake. ${ }^{6,7}$ Calorie restriction or weight loss were not intervention targets. Dietary group participants received 18 group sessions led by centrally trained certified nutritionists in year 1 and quarterly maintenance sessions throughout the dietary intervention period. Comparison group participants received written diet-related education materials. Participants provided a 4-day food record at baseline. In addition to a screening FFQ, FFQs were obtained after 1 year and thereafter from approximately one third of participants yearly in a rotating sample. Postintervention dietary intake was also based on 24-hour dietary recall obtained in a subset of 1,311 reconsenting participants. Information on adjuvant chemotherapy and radiation therapy was based on Medicare findings, whereas adjuvant endocrine therapy use was based on self-report.
Participants with incident breast cancer continued to participate in subsequent dietary group meetings and activities. As such, dietary group participants diagnosed with breast cancer in the first year after random assignment would have approximately 1 year of nutritionist contact before and 7 years of contact after diagnosis. In contrast, participants diagnosed with breast cancer in year 8 after entry would have had more contact before and less after diagnosis. The protocol-specified trial completion date of March 31, 2005, was the end of the 8.5-year dietary intervention period when contact with study nutritionists ended. Subsequent outcome assessment required reconsent obtained from $84 \%$ versus $81 \%$ of surviving comparison and dietary group participants, respectively, for follow-up through 2010 and $86 \%$ of surviving participants from both groups for additional follow-up.

Outcome ascertainment was at 6-month intervals throughout the intervention period, with subsequent updates annually. Initial breast cancer self-reports were confirmed after medical record review by clinical center physician-adjudicators. Final adjudication was performed centrally at the Clinical Coordinating Center. ${ }^{8}$ Human epidermal growth factor receptor 2, ER, and PR status was determined at local laboratories. Cause of death was determined by centralized medical record or death certificate review and, in some cases, by report from participant relatives. All adjudicators were blind to random assignment. National Death Index queries completed through 2013 provided additional survival information regardless of reconsent status.

\section{Statistical Analysis}

The current study primary analysis end points, annualized rates of deaths as a result of and after breast cancer, during the dietary intervention period and cumulatively throughout follow-up were assessed by randomly assigned group by dividing the number of events by the corresponding person-time in each period. Incidence curves were generated, and HRs, 95\% CIs, and $P$ values were calculated from Cox proportional hazards regression models stratified by age at random assignment, random assignment status in the WHI hormone trials, and study period (time dependent). Analyses of deaths as a result of and after breast cancer included all 48,835 study participants measured from random assignment. The study design and the two analysis periods are outlined in Figure 1.

Because dietary effects in nine subgroups were investigated, fewer than one statistically significant interaction was expected by chance alone. The Kaplan-Meier method was used for graphical presentation of death rates. The amount of the dietary effect on death after breast cancer that

WHI Dietary Modification Randomized Controlled Primary Breast Cancer Prevention Clinical Trial Analysis Plan

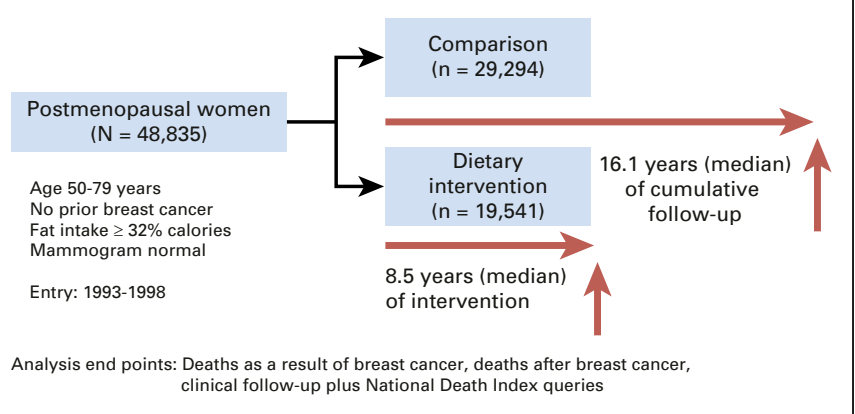

Fig 1. Participant flow for analyses of deaths as a result of and after breast cancer during the 8.3- and 8.5-year (mean and median) dietary intervention period and during the 16.5- and 16.1-year (mean and median) cumulative follow-up period in analyses that incorporated all 48,835 randomly assigned participants. Vertical arrows indicate the span of conducted National Death Index searches. WHI, Women's Health Initiative. 
could be explained by its effect on PR status was assessed by the proportional reduction of the HR, relative to the null, after controlling for PR status. All statistical tests were two-sided. Analyses were performed with SAS 9.4 software (SAS Institute, Cary, NC). The current report updates, with long-term follow-up, dietary modification influence on deaths as a result of breast cancer and provides, for the first time in our knowledge, findings on deaths after breast cancer. These survival analyses were not protocol prespecified but provide the final and most accurate assessment of study findings.

\section{RESULTS}

Although no ongoing contact with study nutritionists occurred during postintervention, the percent energy from fat remained modestly lower in the dietary group for several years. ${ }^{3}$ The participant flow for those diagnosed with breast cancer during the dietary intervention period and throughout the cumulative followup period is outlined in Appendix Figure A1 (online only).

For a subset of participants $(n=1,764)$ with breast cancer diagnosed during the dietary intervention period, selected intervention-related characteristics are outlined at baseline and serially in Appendix Table A1 (online only). Dietary intakes by randomly assigned group at baseline and 1 year in participants with breast cancer were similar to those in the overall study population, and significant differences remained between groups after breast cancer diagnosis for changes in percent energy from fat $(-11.5 \% v-1.5 \% ; P<.001)$ and intake of fruits, vegetables, and grains $(P<.001)$ on the basis of the first FFQ obtained 1.6 years (mean) after diagnosis. Recreational physical activity after breast cancer, available in a subset of 330 participants, was 12.8 versus 10.8 MET-hours/week (mean) for the dietary and comparison groups, respectively $(P=.41)$. Physical activity was not assessed postintervention.

As reported previously, during the 8.5-year dietary intervention period, $8 \%$ fewer breast cancers were observed in the dietary group. ${ }^{1,3}$ In the current report, of all 3,030 breast cancers diagnosed throughout the 16.1 years of cumulative follow-up, the HR for breast cancer incidence was 0.97 (95\% CI, 0.90 to 1.04; Fig 1). Most breast cancer characteristics were balanced between randomly assigned groups. Similar to findings during the dietary intervention period, ${ }^{1}$ a larger fraction of cancers in the dietary group were $\mathrm{ER}+$ and $\mathrm{PR}-(P=.05$; Table 1$)$.

During the 8.5 years of dietary intervention, with 1,764 incident breast cancers, fewer deaths occurred as a result of breast cancer in the dietary group, which was not statistically significant (27 deaths [0.016\% per year] $v 61$ deaths [0.024\% per year]; HR, $0.67 ; 95 \%$ CI, 0.43 to $1.06 ; P=.08$; Figs 2 and $3 \mathrm{~A}$ ). During the same period, deaths after breast cancer $(\mathrm{n}=134)$ were significantly reduced (40 deaths [0.025\% per year] $v 94$ deaths [0.038\% per year]; HR, $0.65 ; 95 \% \mathrm{CI}, 0.45$ to $0.94 ; P=.02)$ by the dietary intervention (Fig 3B). During the 16.1 years of cumulative followup with 3,030 incident breast cancers, somewhat fewer deaths occurred as a result of breast cancer in the dietary group (111 deaths $[0.035 \%$ per year $] v 185$ deaths $[0.039 \%$ per year $]$; HR, 0.91 ; 95\% CI, 0.72 to 1.15; Fig 4A), and deaths after breast cancer were significantly reduced (234 deaths $[0.085 \%$ per year] $v 443$ deaths

\begin{tabular}{|c|c|c|c|c|c|}
\hline \multirow[b]{2}{*}{ Characteristic } & \multicolumn{2}{|c|}{$\begin{array}{l}\text { Intervention } \\
(\mathrm{n}=1,176) \\
\end{array}$} & \multicolumn{2}{|c|}{$\begin{array}{l}\text { Comparison } \\
(\mathrm{n}=1,854)\end{array}$} & \multirow[b]{2}{*}{$P^{*}$} \\
\hline & No. & $\%$ & No. & $\%$ & \\
\hline Histology & & & & & .89 \\
\hline Ductal & 752 & 64.4 & 1,202 & 65.3 & \\
\hline Lobular & 116 & 9.9 & 177 & 9.6 & \\
\hline Ductal and lobular & 155 & 13.3 & 248 & 13.5 & \\
\hline Other & 145 & 12.4 & 213 & 11.6 & \\
\hline ER status & & & & & .59 \\
\hline Positive & 945 & 85.0 & 1,499 & 85.7 & \\
\hline Negative & 167 & 15.0 & 250 & 14.3 & \\
\hline PR status & & & & & .08 \\
\hline Positive & 812 & 73.9 & 1,226 & 70.9 & \\
\hline Negative & 287 & 26.1 & 504 & 29.1 & \\
\hline ER/PR status & & & & & .05 \\
\hline $\mathrm{ER}+, \mathrm{PR}+$ & 793 & 73.6 & 1,200 & 70.5 & \\
\hline $\mathrm{ER}+, \mathrm{PR}-$ & 141 & 13.1 & 281 & 16.5 & \\
\hline ER-, PR- & 144 & 13.4 & 221 & 13.0 & \\
\hline HER2 overexpression & 146 & 15.3 & 202 & 13.7 & .28 \\
\hline Triple-negative tumor & 76 & 8.1 & 133 & 9.1 & .37 \\
\hline Stage & & & & & .90 \\
\hline Local & 870 & 75.5 & 1,371 & 75.2 & \\
\hline Regional or distant & 283 & 24.5 & 451 & 24.8 & \\
\hline Grading & & & & & .40 \\
\hline Well differentiated & 309 & 29.0 & 486 & 28.7 & \\
\hline Moderately differentiated & 454 & 42.6 & 772 & 45.7 & \\
\hline Poorly differentiated & 302 & 28.4 & 433 & 25.6 & \\
\hline Tumor size, cm & & & & & .98 \\
\hline$<1$ & 307 & 28.6 & 504 & 29.6 & \\
\hline 1 to $<2$ & 482 & 44.8 & 727 & 42.7 & \\
\hline$\geq 2$ & 286 & 26.6 & 472 & 27.7 & \\
\hline Positive lymph nodes & & & & & .98 \\
\hline None & 789 & 75.5 & 1,252 & 75.8 & \\
\hline 1 to 3 & 195 & 18.7 & 296 & 17.9 & \\
\hline$\geq 4$ & 61 & 5.8 & 103 & 6.2 & \\
\hline
\end{tabular}

Abbreviations: ER, estrogen receptor; HER2, human epidermal growth factor receptor 2; PR, progesterone receptor.

* $P$ values are based on $\chi^{2}$ tests of association for most tumor characteristics. Trend tests were used for ordinal tumor characteristics: grading, tumor size, and positive lymph nodes.

[0.11\% per year]; HR, $0.82 ; 95 \% \mathrm{CI}, 0.70$ to $0.96 ; P=.01)$ in the dietary group (Fig 4B).

The HR for deaths after breast cancer was unchanged by the addition of baseline weight and time-dependent weight change to the analysis. Analyses that added a time-dependent variable for PR status resulted in an HR of 0.87 (95\% CI, 0.74 to 1.02) during the cumulative follow-up period, which corresponded to a $29 \%$ explanation of the benefit observed in the unadjusted analyses (Figs $3 \mathrm{~B}$ and $4 \mathrm{~B})$.

Breast cancer therapy information from Medicare was available in 1,403 of 3,030 participants; no significant differences between the dietary and comparison groups were seen for radiation therapy $(64 \% \vee 65 \%$, respectively) or adjuvant chemotherapy (25\% v 27\%, respectively). Endocrine adjuvant therapy, which was based on self-report $(n=673)$, also was similar in the randomly assigned groups (71\% v 68\% users, respectively).

Two of nine subgroups examined for dietary modification influence on deaths after breast cancer were significant at the .05 level 


\begin{tabular}{|c|c|c|c|c|c|c|c|c|c|c|}
\hline & $\begin{array}{l}\text { Intervention } \\
\text { No. (\%) }\end{array}$ & $\begin{array}{c}\text { Comparison } \\
\text { No. }(\%)\end{array}$ & HR & $(95 \% \mathrm{Cl})$ & $P$ & & & & & \\
\hline \multicolumn{11}{|l|}{ Invasive breast cancer } \\
\hline Intervention period & $671(0.42)$ & $1,093(0.46)$ & 0.92 & 0.84 to 1.01 & .09 & & & & & \\
\hline Cumulative: intervention + postintervention periods & $1,176(0.44)$ & $1,854(0.46)$ & 0.97 & 0.90 to 1.04 & .34 & & & - & & \\
\hline \multicolumn{11}{|l|}{ Death as a result of breast cancer } \\
\hline Intervention period & $27(0.016)$ & $61(0.024)$ & 0.67 & 0.43 to 1.06 & .08 & $\leftarrow$ & & & & \\
\hline Cumulative: intervention + postintervention periods & $111(0.035)$ & $185(0.039)$ & 0.91 & 0.72 to 1.15 & .41 & & & & & \\
\hline \multicolumn{11}{|l|}{ Death after breast cancer } \\
\hline Intervention period & $40(0.025)$ & $94(0.038)$ & 0.65 & 0.45 to 0.94 & .02 & $\leftarrow$ & & & & \\
\hline Cumulative: intervention + postintervention periods* & $174(0.10)$ & $342(0.14)$ & 0.81 & 0.67 to 0.97 & .02 & & & & & \\
\hline \multirow[t]{3}{*}{ Cumulative: intervention + postintervention periods $\uparrow$} & $234(0.085)$ & $443(0.11)$ & 0.82 & 0.70 to 0.96 & .01 & & & & & \\
\hline & & & & & & 0.50 & 0.75 & 1.00 & 1.33 & 2.00 \\
\hline & & & & & & \multicolumn{5}{|c|}{ Favors intervention } \\
\hline
\end{tabular}

Fig 2. Breast cancer results by study period. Forest plot and summary statistics of the dietary modification influence on invasive breast cancer incidence, deaths as a result of breast cancer, and deaths after breast cancer by study period (dietary intervention period, 8.5 years; cumulative follow-up [intervention + postintervention] period, 16.1 years). ${ }^{*}$ ) Includes deaths as a result of any cause that occurred after breast cancer diagnosis during the intervention period only. (t) Includes deaths as a result of any cause that occurred after breast cancer diagnosis during the intervention or postintervention period.

(waist circumference and percent energy from fat [on the basis of case-only analysis in participants with a 4-day food record baseline assessment]; Fig 5). In participants with breast cancer, information on cause of death was available in 668 of 677 . Breast cancer was the most common cause $(38 \% ; \mathrm{n}=257)$ followed by other cancers $(20 \%$; $\mathrm{n}=132)$ and cardiovascular disease $(18 \% ; \mathrm{n}=120)$.

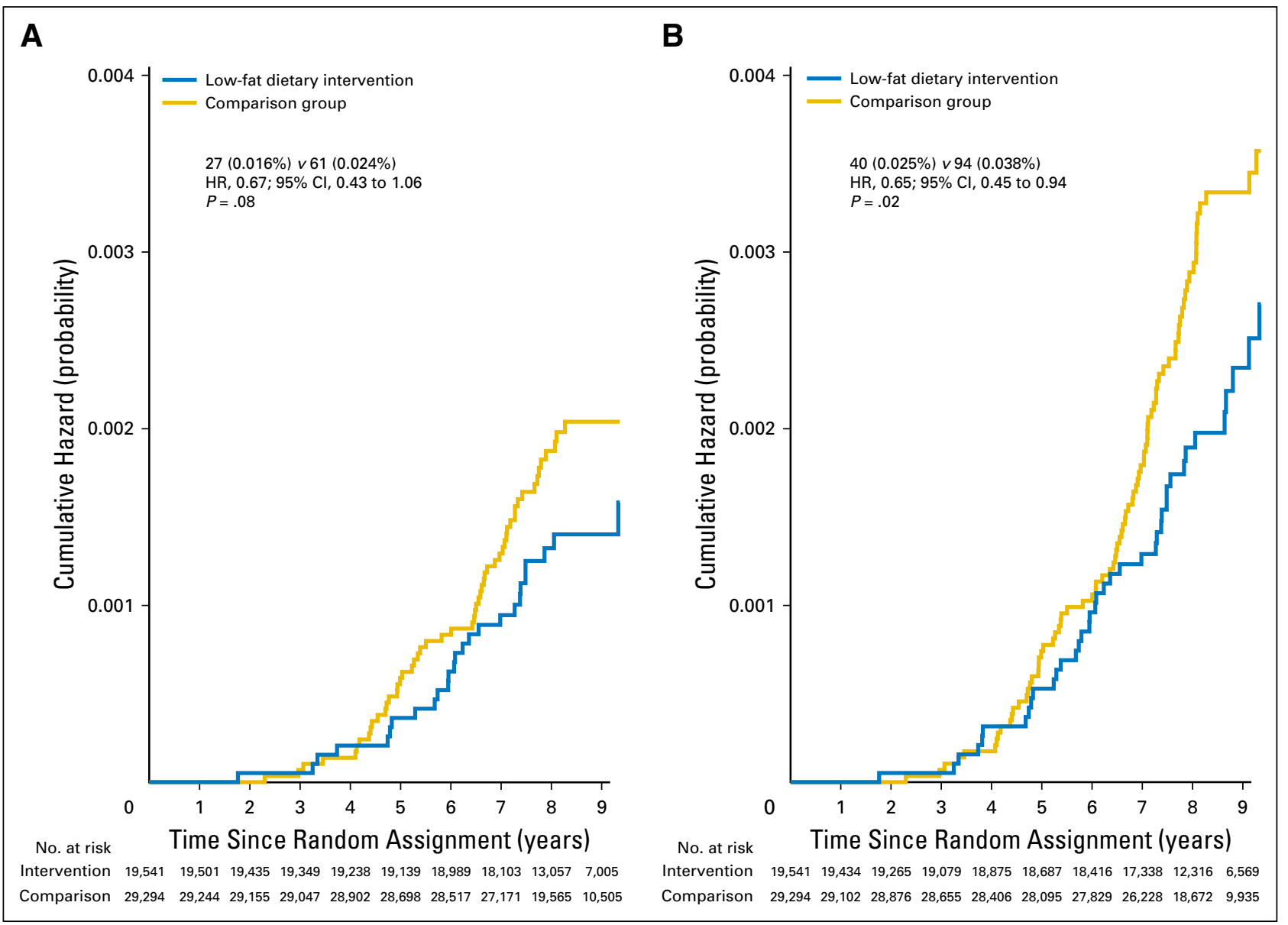

Fig 3. Dietary modification influence on deaths as a result of and after breast cancer during the 8.5-year (median) dietary intervention period. (A) Kaplan-Meier cumulative hazard estimates for death as a result of breast cancer (breast cancer followed by death attributed to the cancer) during the 8.5-year (median) dietary intervention period and (B) Kaplan-Meier cumulative hazard estimates for death after breast cancer (breast cancer followed by death as a result of any cause) during the dietary intervention period among all 48,835 trial participants, with 1,764 breast cancers measured since random assignment. Summary statistics are from a Cox proportional hazards regression model stratified by age-group and random assignment in the hormone therapy trials. The $P$ value corresponds to a two-sided score (log-rank test). HR, hazard ratio. 

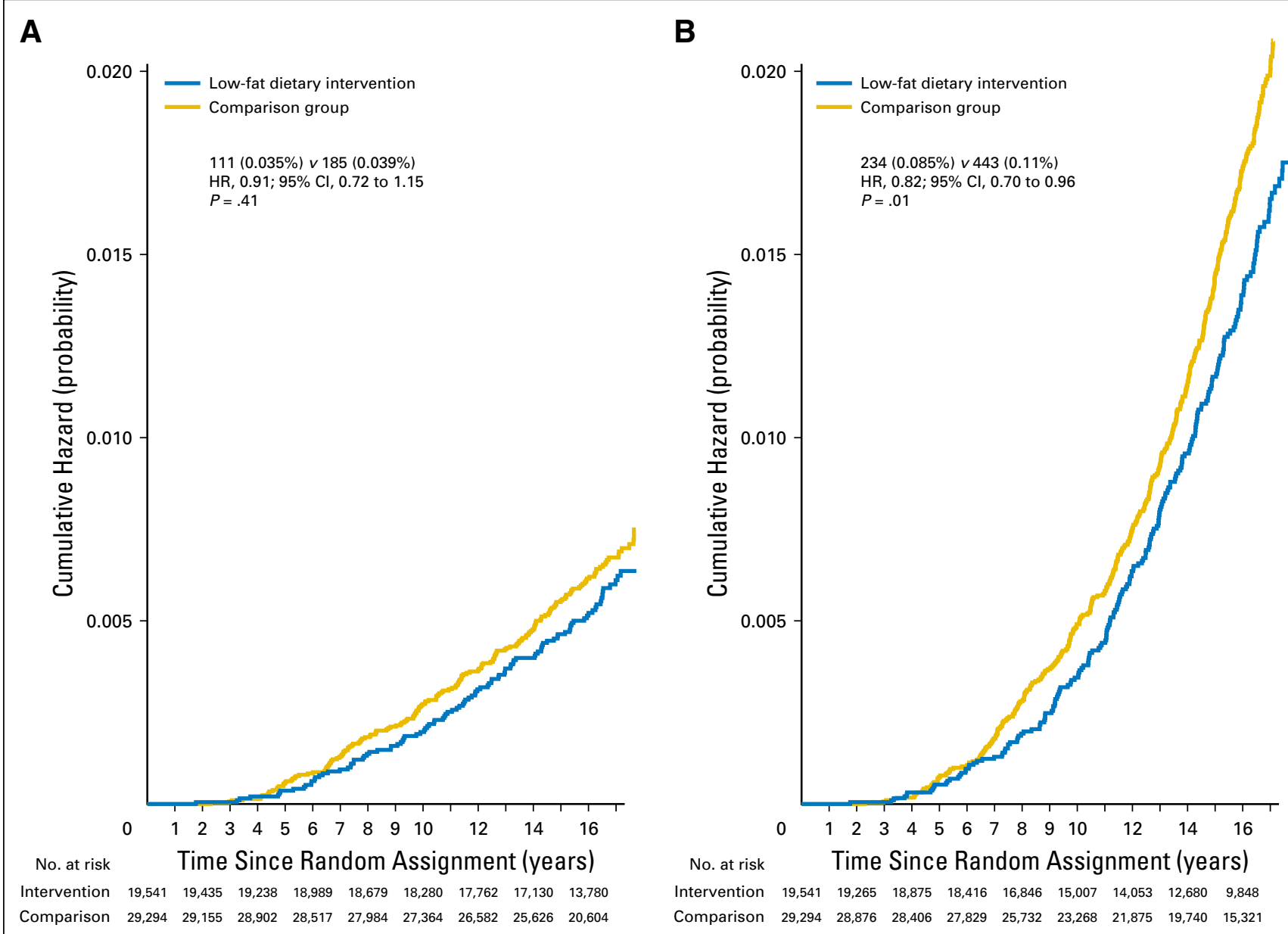

Fig 4. Dietary modification influence on deaths as a result of and after breast cancer during the 16.1-year (median) cumulative follow-up. (A) Kaplan-Meier cumulative

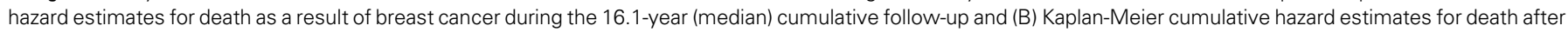

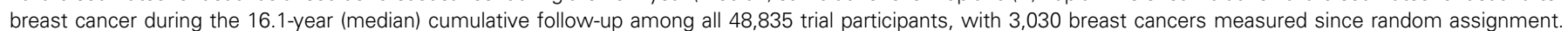

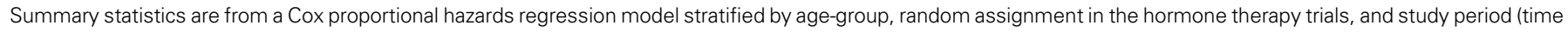
dependent). The $P$ value corresponds to a two-sided score (log-rank test). HR, hazard ratio.

\section{DISCUSSION}

With long-term follow-up of the WHI DM trial, deaths after breast cancer were significantly reduced in the dietary group both during the dietary intervention period and throughout the 16.1-year cumulative follow-up period. The lower risk of poor prognosis, ER+, PR- breast cancers ${ }^{10}$ in the dietary group contributed to the favorable dietary effect on death after breast cancer. The findings on deaths as a result of and after breast cancer presented here arose from standard intention-to-treat comparisons between randomly assigned groups in analyses that included all 48,835 participants. WHI DM is a primary prevention trial; however, in adjuvant breast cancer trials, death as a result of all causes measured from diagnosis (breast cancer overall survival) is considered an important clinical end point. ${ }^{11,12}$ An additional factor that potentially influenced deaths after breast cancer could be a favorable dietary influence on mortality as a result of other causes, including cardiovascular disease.

Although fewer deaths occurred as a result of breast cancer in the dietary group, the difference was not statistically significant and perhaps was limited by event number. However, the HR for dietary influence on deaths as a result of breast cancer during the dietary intervention period was comparable to that seen for deaths after breast cancer (HR, 0.67 and 0.65 , respectively). In addition, accurate determination of cause of death after breast cancer can be difficult given the potential interaction between common comorbidities and cancer therapies, such as cardiac disease after anthracycline, trastuzumab, or radiation therapy. Thus, the actual breast cancer mortality rate likely lies between the attributed rate, on the basis of medical record review, and the rate that considered all deaths after breast cancer.

The subgroup analyses identified postmenopausal women as more likely to benefit from the dietary intervention. An intervention effect on deaths after breast cancer was more likely if a woman had either a lifestyle ( $\geq 36.8 \%$ energy from fat) or a consequence of lifestyle ( $\geq 88$ waist circumference) that has been associated with adverse breast cancer outcome before dietary modification. ${ }^{13}$ Because subgroup analysis identified no benefit on deaths after breast cancer for dietary group participants, with $<27.9 \%$ calories from fat at entry, a notional threshold for effect may be $>28 \%$ calories from fat, which is not far from the current average US intake of postmenopausal women of $33 \% .{ }^{14} \mathrm{~A}$ modest reduction in fat intake with minimal weight loss represents an easily achievable goal by many. 


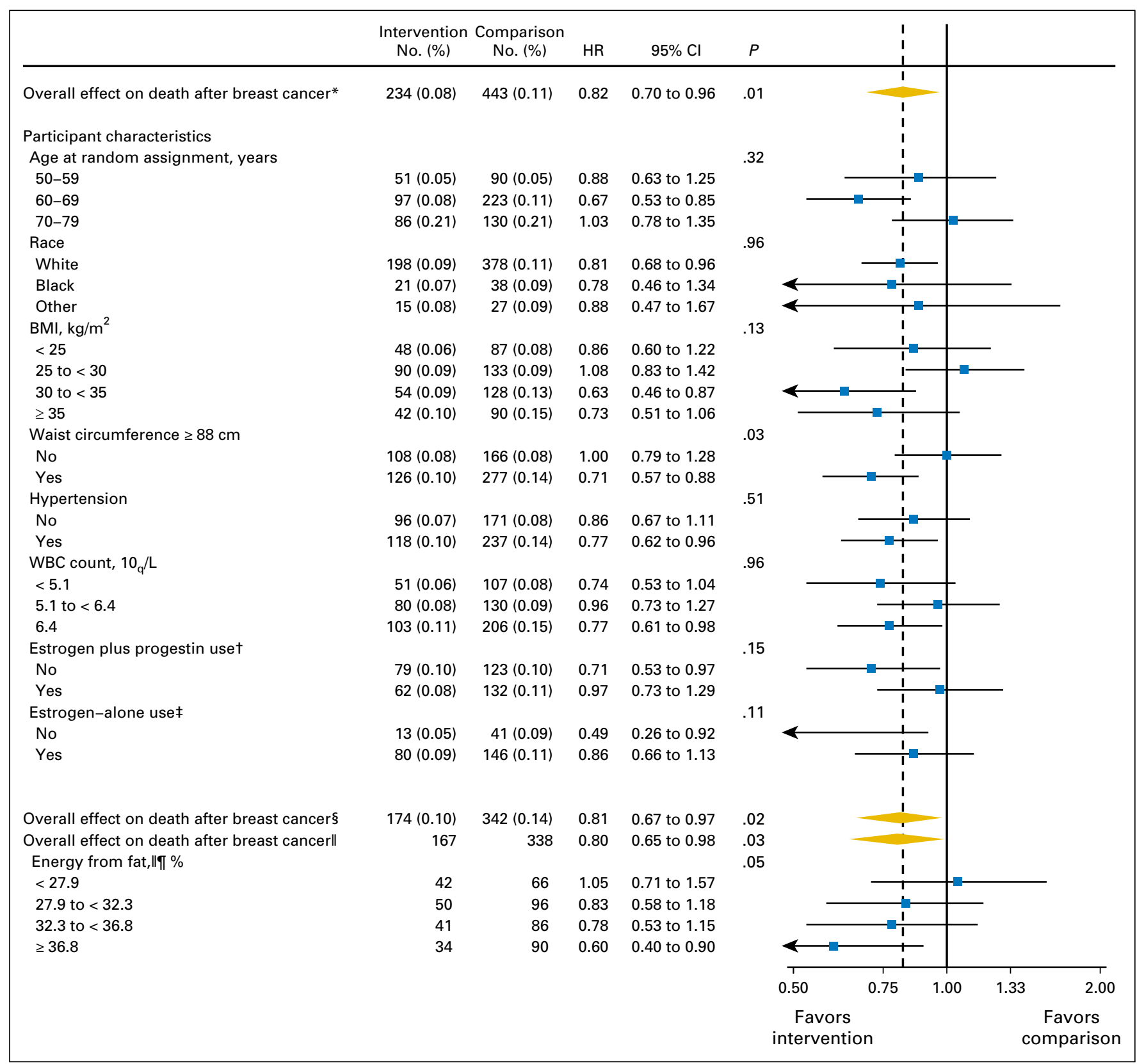

Fig 5. Subgroup analysis by a forest plot of hazard ratios (HRs), dietary intervention versus comparison, for death after breast cancer by select subgroups of participants with an invasive breast cancer diagnosis that occurred anytime throughout the cumulative follow-up period. Annualized percentages are shown for all subgroups except percent energy from fat, where the numbers of deaths with 4-day food records were reported. Because of expense, baseline food records were scored for only breast cancers that occurred during the intervention period. For this analysis HRs were estimated in a case-only analysis by using logistic regression of random assignment stratified by the four ordinal groups of dietary fat. For rare outcomes (eg, $<5 \%$ incidence during study follow-up), case-only analyses provided HRs and corresponding $95 \%$ $\mathrm{Cls}$ that were essentially equivalent to those that would arise if the subgroups were available for the entire randomly assigned cohort. To illustrate, the corresponding caseonly estimate for the overall effect on death after breast cancer $(\mathrm{HR}, 0.80)$ and the usual Cox proportional hazards regression estimate that includes deaths as a result of any cause that occurred after breast cancer diagnosis during the intervention period only (HR, 0.81) are provided. $P$ values correspond to a test of the interaction between randomly assigned group and subgroup. $\left(^{*}\right)$ Includes deaths as a result of any cause that occurred after breast cancer diagnosis during the intervention or postintervention periods. $(\dagger)$ Among participants who did not report a prior hysterectomy. Ever used estrogen plus progestin includes random assignment into the Women's Health Initiative conjugated equine estrogens plus medroxyprogesterone acetate trial. (¥) Among participants who reported a prior hysterectomy. Ever used estrogen alone includes random assignment into Women's Health Initiative conjugated equine estrogens alone trial. §lncludes deaths as a result of any cause that occurred after breast cancer diagnosis during the intervention period only. \|Includes deaths as a result of any cause that occurred after breast cancer diagnosis during the intervention period only. Estimates were strictly based on participants who had baseline 4-day food records available (98\%; $505=167+338$ of $516=174+342)$. (I) Baseline quartiles on the basis of 4 -day food records of a $4.6 \%$ random subsample. BMI, body mass index.

A substantial number of observational studies have addressed the issue of dietary intake and breast cancer incidence and outcome, but the findings were mixed, ${ }^{15-17}$ with both negative ${ }^{18}$ and positive ${ }^{19}$ findings that perhaps reflected observational study limitations. These limitations include dietary intake measurement error, limited variation in dietary intake within study populations, and common reliance 
on a single dietary intake made before diagnosis. The uncertainty raised by the observational study findings provided a major impetus to the conduct of the current randomized clinical trial.

The WHI low-fat dietary program reduced fat and increased fruit and vegetable intake, changes that potentially influence breast cancer, ${ }^{20-22}$ and their effects on study findings cannot be separated. Although adjustment for weight change during the intervention did not attenuate the dietary effect on death after breast cancer, the WHI DM trial evaluated a low-fat eating pattern, not the influence of any individual factor on study outcomes. With respect to other lifestyle factors, when measured in a subgroup, physical activity did not significantly differ between randomly assigned groups.

At entry, women in the WHI DM trial had no prior breast cancer and normal screening mammogram. Nonetheless, results from a modeling exercise suggest that the WHI dietary intervention may have primarily affected undiagnosed preclinical breast cancers. The model $^{23}$ incorporates cancer doubling time, mammography detection threshold, and subclinical breast cancer prevalence. When applied to the WHI estrogen plus progestin trial, which entered a similarly aged population, after a mean 5.6 years of follow-up, 94\% of the breast cancers were estimated to be preclinical cancers, with only $6 \%$ de novo cases. ${ }^{23}$

In the WHI DM trial, after 16.1 years of follow-up, no overall reduction in breast cancer incidence was seen, but a smaller fraction of cancers in the dietary group were $\mathrm{ER}+, \mathrm{PR}-$, which explains $29 \%$ of the difference in deaths after breast cancer. Overall, $9 \%$ fewer deaths occurred as a result of breast cancer $(n=296$; $P=.41)$, and $18 \%$ fewer deaths occurred after breast cancer $(n=677$; $P=.01)$ in the dietary group. One interpretation could be that dietary intervention-reduced breast cancers are more likely to be fatal and favorably influence other causes of death that may or may not have been specific to the cancer therapy experience of patients with breast cancer. Because the WHI low-fat dietary intervention had at least short-term effects of lowering systolic blood pressure, reducing metabolic syndrome risk, and lowering cholesterol-targeted and hypertension medicine, ${ }^{24,25}$ it could have had an influence on other chronic diseases.

The WHI dietary intervention findings can be compared with three randomized primary breast cancer prevention trials that evaluated tamoxifen. $^{26-28}$ In the P-1 trial in 13,388 participants, after a median 7 years of follow-up, tamoxifen reduced breast cancer incidence by $43 \%$, but $10 \%$ more deaths occurred after breast cancer in the tamoxifen group ( $n=240$; not significant). ${ }^{26}$ In the Royal Marsden Prevention Trial in 2,471 participants, after a median 13 years of follow-up, breast cancer incidence was somewhat lower in the tamoxifen group (22\% lower, $P=.1)$, with 27 deaths after breast cancer in each randomly assigned group. ${ }^{27}$ In the International Breast Cancer Intervention Trial 1 in 7,154 participants, after a median 16 years follow-up, tamoxifen reduced breast cancer incidence by $29 \%(P<.001)$, but $19 \%$ more deaths occurred as a result of breast cancer $(\mathrm{n}=57 ; P=.8)$ and $10 \%$ more deaths after breast cancer $(\mathrm{n}=389 ; P=0.4)$ in the tamoxifen group. ${ }^{28}$ Favorable effects on mortality may emerge from these trials with longer follow-up, and differences in study populations and conduct exist compared with the WHI DM trial. However, one interpretation of the current tamoxifen findings could be that tamoxifen reduced breast cancers that were less likely to be fatal and did not favorably influence other causes of death. ${ }^{29}$

To our knowledge, only two other full-scale randomized trials examined dietary change and breast cancer incidence. A low-fat intervention was evaluated in 4,690 women with high breast density but found no influence on breast cancer incidence (HR, 1.19; 95\% CI, 0.91 to 1.55$).{ }^{30}$ Compared with the WHI DM, the study population was younger (mean age, 47 years), commonly premenopausal, and had lower a lower body mass index (mean, $23.3 \mathrm{~kg} / \mathrm{m}^{2}$ ). In the Prevención con Dieta Mediterránea trial, 4,152 postmenopausal women at high cardiovascular risk were randomly assigned to two Mediterranean diets or a control condition (advised to reduce dietary fat). As a secondary end point, breast cancer incidence $(n=35)$ was significantly lower in one Mediterranean diet compared with the control condition. However, control group counseling was limited, and participants did not materially reduce fat intake. ${ }^{31}$

Strengths of the current study include the randomized design, a large population of 48,835 postmenopausal women, 3,030 incident breast cancers confirmed by medical record review and 677 deaths after breast cancer, serial screening mammography, and long-term follow-up. National Death Index supplemental follow-up provided additional survival information independent of reconsent status. Although no established biomarkers existed for dietary fat intake, the sustained lower body weight in the dietary group supports dietary intake differences between randomly assigned groups. Study limitations are associated with post hoc analyses and lack of comprehensive breast cancer therapy information.

In summary, women randomly assigned to a low-fat dietary pattern had a nonsignificantly lower risk of death as a result of breast cancer and a significantly reduced risk of death after breast cancer. Future studies of other lifestyle interventions on breast cancer incidence and outcome could incorporate some form of a low-fat dietary pattern as a base.

\section{AUTHORS' DISCLOSURES OF POTENTIAL CONFLICTS} OF INTEREST

Disclosures provided by the authors are available with this article at jco.org.

\section{AUTHOR CONTRIBUTIONS}

Conception and design: Rowan T. Chlebowski, Aaron K. Aragaki, Garnet L. Anderson, Ross L. Prentice

Financial support: Rowan T. Chlebowski

Administrative support: Rowan T. Chlebowski, Garnet L. Anderson, Barbara V. Howard, Ross L. Prentice

Provision of study materials or patients: Rowan T. Chlebowski, Garnet L. Anderson, Cynthia A. Thomson, JoAnn E. Manson, Dorothy Lane, Ross L. Prentice

Collection and assembly of data: Rowan T. Chlebowski, Garnet L. Anderson, JoAnn E. Manson, Barbara V. Howard, Ross L. Prentice

Data analysis and interpretation: All authors

Manuscript writing: All authors

Final approval of manuscript: All authors

Accountable for all aspects of the work: All authors 


\section{REFERENCES}

1. Prentice RL, Caan B, Chlebowski RT, et al: Low-fat dietary pattern and risk of invasive breast cancer: The Women's Health Initiative randomized controlled dietary modification trial. JAMA 295: 629-642, 2006

2. Howard BV, Manson JE, Stefanick ML, et al: Low-fat dietary pattern and weight change over 7 years: The Women's Health Initiative Dietary Modification trial. JAMA 295:39-49, 2006

3. Thomson CA, Van Horn L, Caan BJ, et al: Cancer incidence and mortality during the intervention and postintervention periods of the Women's Health Initiative Dietary Modification trial. Cancer Epidemiol Biomarkers Prev 23:2924-2935, 2014

4. The Women's Health Initiative Study Group: Design of the Women's Health Initiative clinical trial and observational study. Control Clin Trials 19: 61-109, 1998

5. Anderson GL, Manson J, Wallace R, et al: Implementation of the Women's Health Initiative study design. Ann Epidemiol 13S5-S17, 2003 (suppl 9)

6. Patterson RE, Kristal $A R$, Tinker $L F$, et al: Measurement characteristics of the Women's Health Initiative food frequency questionnaire. Ann Epidemiol 9:178-187, 1999

7. Tinker LF, Burrows ER, Henry $H$, et al: Women's Health Initiative: Overview of the nutrition component, in Kummel DA, Kris-Atherton PM (eds): Nutrition in Women's Health. Gaithersburg, MD, Aspen, 1996, pp 510-542

8. National Cancer Institute: Surveillance, Epidemiology, and End Results Program. https://seer. cancer.gov

9. Reference deleted.

10. Cancello G, Maisonneuve P, Rotmensz N, et al: Progesterone receptor loss identifies luminal $B$ breast cancer subgroups at higher risk of relapse. Ann Oncol 24:661-668, 2013
11. Colleoni M, Giobbie-Hurder A, Regan MM, et al: Analyses adjusting for selective crossover show improved overall survival with adjuvant letrozole compared with tamoxifen in the BIG 1-98 study. J Clin Oncol 29:1117-1124, 2011

12. Perez EA, Romond EH, Suman VJ, et al: Trastuzumab plus adjuvant chemotherapy for human epidermal growth factor receptor 2-positive breast cancer: Planned joint analysis of overall survival from NSABP B-31 and NCCTG N9831. J Clin Oncol 32: 3744-3752, 2014

13. Jiralerspong S, Goodwin PJ: Obesity and breast cancer prognosis: Evidence, challenges, and opportunities. J Clin Oncol 34:4203-4216, 2016

14. Centers for Disease Control and Prevention; National Center for Health Statistics: Diet/nutrition. https://www.cdc.gov/nchs/fastats/diet.htm

15. Chlebowski RT: Nutrition and physical activity influence on breast cancer incidence and outcome. Breast 22: S30-S37, 2013 (suppl 2)

16. Brennan SF, Woodside JV, Lunny PM, et al: Dietary fat and breast cancer mortality: A systematic review and meta-analysis. Crit Rev Food Sci Nutr 57: 1999-2008, 2017

17. Cao $Y$, Hou L, Wang W: Dietary total fat and fatty acids intake, serum fatty acids and risk of breast cancer: A meta-analysis of prospective cohort studies. Int J Cancer 138:1894-1904, 2016

18. Boeke $C E$, Eliassen $A H$, Chen WY, et al: Dietary fat intake in relation to lethal breast cancer in two large prospective cohort studies. Breast Cancer Res Treat 146:383-392, 2014

19. Kroenke $\mathrm{CH}$, Kwan $\mathrm{ML}$, Sweeney $\mathrm{C}$, et al: High- and low-fat dairy intake, recurrence, and mortality after breast cancer diagnosis. J Natl Cancer Inst 105:616-623, 2013

20. Chlebowski RT, Aiello E, McTiernan A: Weight loss in breast cancer patient management. $\mathrm{J}$ Clin Oncol 20:1128-1143, 2002

21. Chan DS, Vieira AR, Aune $D$, et al: Body mass index and survival in women with breast cancer- systematic literature review and meta-analysis of 82 follow-up studies. Ann Oncol 25:1901-1914, 2014
22. Ligibel JA, Chlebowski RT: Lifestyle issues in breast cancer survivors, in Harris JR, Lippman ME, Morrow M, et al (eds): Diseases of the Breast (ed 5). Philadelphia, PA, Lippincott Williams \&Wilkins, 2014, pp 676-681

23. Santen RJ, Yue W, Heitjan DF: Occult breast tumor reservoir: Biological properties and clinical significance. Horm Cancer 4:195-207, 2013

24. Neuhouser ML, Howard B, Lu J, et al: A low-fat dietary pattern and risk of metabolic syndrome in postmenopausal women: The Women's Health Initiative. Metabolism 61:1572-1581, 2012

25. Allison MA, Aragaki AK, Ray RM, et al: $A$ randomized trial of a low-fat dietary intervention on blood pressure and hypertension: Tertiary analyses of the WHI Dietary Modification trial. Am J Hypertens 29:959-968, 2016

26. Fisher $B$, Costantino JP, Wickerham DL, et al: Tamoxifen for the prevention of breast cancer: Current status of the National Surgical Adjuvant Breast and Bowel Project P-1 study. J Natl Cancer Inst 97: 1652-1662, 2005

27. Powles TJ, Ashley S, Tidy A, et al: Twenty-year follow-up of the Royal Marsden randomized, doubleblinded tamoxifen breast cancer prevention trial. J Natl Cancer Inst 99:283-290, 2007

28. Cuzick J, Sestak I, Cawthorn S, et al: Tamoxifen for prevention of breast cancer: Extended long-term follow-up of the IBIS-I breast cancer prevention trial. Lancet Oncol 16:67-75, 2015

29. Chlebowski RT: IBIS-I tamoxifen update: Maturity brings questions. Lancet Oncol 16:7-9, 2015

30. Martin LJ, Li Q, Melnichouk O, et al: A randomized trial of dietary intervention for breast cancer prevention. Cancer Res 71:123-133, 2011

31. Toledo E, Salas-Salvadó J, Donat-Vargas C et al: Mediterranean diet and invasive breast cancer risk among women at high cardiovascular risk in the PREDIMED trial: A randomized clinical trial. JAMA Intern Med 175:1752-1760, 2015

Affiliations
Rowan T. Chlebowski, Los Angeles Biomedical Research Institute, Torrance; Lihong Qi, University of California, Davis, Davis, CA; Aaron K. Aragaki, Garnet L. Anderson, Wendy Barrington, and Ross L. Prentice, Fred Hutchinson Cancer Research Center, Seattle, WA; Cynthia A. Thomson, University of Arizona Cancer Center, Tucson, AZ; JoAnn E. Manson, Brigham and Women's Hospital, Boston, MA; Michael S. Simon, Wayne State University, Detroit, MI; Barbara V. Howard, MedStar Health Research Institute; Barbara V. Howard, Georgetown-Howard Universities Center for Clinical and Translational Sciences, Washington, DC; Thomas E. Rohan, Albert Einstein College of Medicine, New York City; Dorothy Lane, Stony Brook University, Stony Brook, NY; Linda Snetselar, University of Iowa, Iowa City, IA; Catherine Womack, Methodist Healthcare; Fridtjof Thomas, University of Tennessee Health Science Center, Memphis, TN; Mara Z. Vitolins, Wake Forest School of Medicine, Winston-Salem, NC; and Lifang Hou, Northwestern University, Chicago, IL.

\section{Support}

Supported by National Heart, Lung, and Blood Institute Grants N01WH22110, 24152, 32100-2, 32105-6, 32108-9, 32111-13, 32115, 32118-32119, 32122, 42107-26, 42129-32, and 44221; American Institute for Cancer Research Grant 30210-01 (to R.T.C.); and National Cancer Institute Grant UM1CA173642 (to R.T.C., G.L.A., and R.L.P.).

\section{Prior Presentation}

Presented in part at the San Antonio Breast Cancer Symposium, San Antonio, TX, December 6-10, 2016, and at the American Association for Cancer Research Annual Meeting, New Orleans, LA, April 16-20, 2016. 


\section{AUTHORS' DISCLOSURES OF POTENTIAL CONFLICTS OF INTEREST}

Low-Fat Dietary Pattern and Breast Cancer Mortality in the Women's Health Initiative Randomized Controlled Trial

The following represents disclosure information provided by authors of this manuscript. All relationships are considered compensated. Relationships are self-held unless noted. I = Immediate Family Member, Inst = My Institution. Relationships may not relate to the subject matter of this manuscript. For more information about ASCO's conflict of interest policy, please refer to www.asco.org/rwc or ascopubs.org/jco/site/ifc.

Rowan T. Chlebowski

Consulting or Advisory Role: Novartis, Genentech, Amgen, Pfizer, AstraZeneca

Speakers' Bureau: Novartis, Genentech

Aaron K. Aragaki

No relationship to disclose

\section{Garnet L. Anderson}

Research Funding: Pfizer (I), Johnson \& Johnson (I), Mars Symbioscience

Cynthia A. Thomson

No relationship to disclose

JoAnn E. Manson

No relationship to disclose

Michael S. Simon

No relationship to disclose

Barbara V. Howard

No relationship to disclose

Thomas E. Rohan

Stock or Other Ownership: MetaStat

Patents, Royalties, Other Intellectual Property: receive royalties on

a patent for tumor microenvironment of metastasis

\section{Linda Snetselar}

No relationship to disclose

Dorothy Lane

No relationship to disclose

Wendy Barrington

No relationship to disclose

Mara Z. Vitolins

No relationship to disclose

Catherine Womack

No relationship to disclose

Lihong Qi

No relationship to disclose

Lifang Hou

No relationship to disclose

Fridtjof Thomas

No relationship to disclose

Ross L. Prentice

No relationship to disclose 


\section{Acknowledgment}

The Women's Health Initiative (WHI) Project Office at National Heart, Lung, and Blood Institute reviewed and approved the final manuscript but played no other role in the preparation of this report. We thank the WHI investigators, staff, and the trial participants for their outstanding dedication and commitment.

\section{Appendix}

\section{Women's Health Initiative Investigators}

Program Office: (National Heart, Lung, and Blood Institute, Bethesda, MD) Jacques Roscoe, Shari Ludlum, Dale Burden, Joan McGowan, Leslie Ford, and Nancy Geller

Clinical Coordinating Center: (Fred Hutchinson Cancer Research Center, Seattle, WA) Garnet Anderson, Ross Prentice, Andrea LaCroix, and Charles Kopperberg)

Investigators and Academic Centers: (Brigham and Women's Hospital, Harvard Medical School, Boston, MA) JoAnn E. Manson, (MedStar Health Research Institute/Howard University, Washington, DC) Barbara V. Howard, (Stanford Prevention Research Center, Stanford, CA) Marcia L. Stefanick, (The Ohio State University, Columbus, OH) Rebecca Jackson, (University of Arizona, Tucson/Phoenix, AZ) Cynthia A. Thompson, (University at Buffalo, Buffalo, NY) Jean Wactawski-Wende, (University of Florida, Gainesville/Jacksonville, FL) Marian Limacher, (University of Iowa, Iowa City/Davenport, IA) Robert Wallace, (University of Pittsburgh, Pittsburgh, PA) Lewis Kuller, (Los Angeles Biomedical Research Institute at Harbor-UCLA Medical Center, Torrance, CA. Now at City of Hope National Medical Center, Duarte, CA) Rowan T. Chlebowski, (Wake Forest University School of Medicine, Winston-Salem, NC) Sally Shumaker

Women's Health Initiative Memory Study: (Wake Forest University School of Medicine, Winston Salem, NC) Sally Shumaker A full list of all the investigators who have contributed to Women's Health Initiative science appears at https://www.whi.org/ researchers/Documents\%20\%20Write\%20a\%20Paper/WHI\%20Investigator\%20Long\%20List.pdf. 


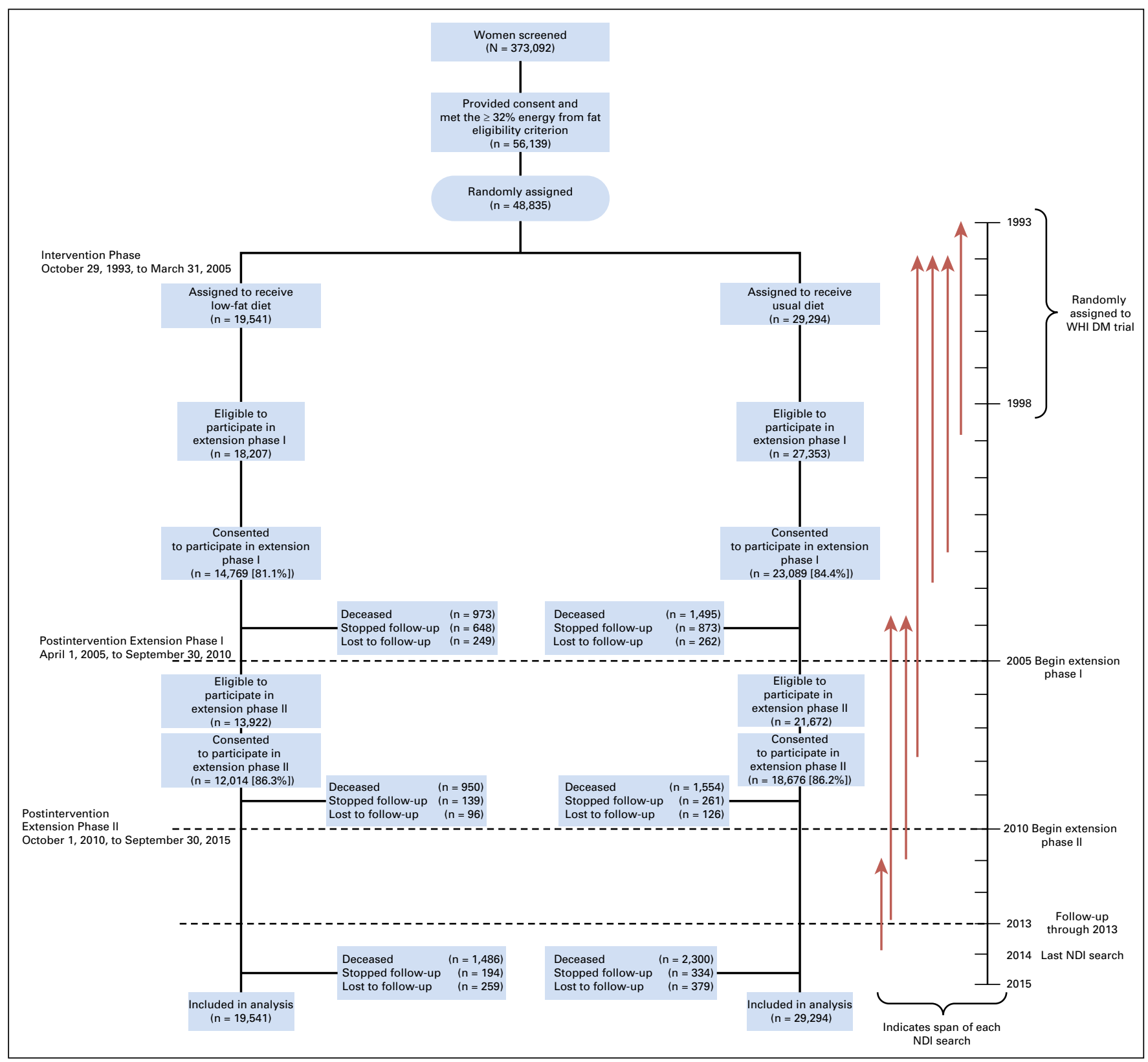

Fig A1. Study design. The arrows identify the two analysis periods (at the end of the 8.3- and 8.5-year [mean and median] dietary intervention period and after 16.5 and 16.1 [mean and median] years of cumulative follow-up). NDI, National Death Index; WHI DM, Women's Health Initiative Dietary Modification. 


\begin{tabular}{|c|c|c|c|c|c|}
\hline Characteristic & \multicolumn{2}{|c|}{$\begin{array}{l}\text { Intervention } \\
(\mathrm{n}=671)\end{array}$} & \multicolumn{2}{|c|}{$\begin{array}{l}\text { Comparison } \\
(\mathrm{n}=1,093)\end{array}$} & $P$ \\
\hline \multicolumn{6}{|l|}{ Baseline } \\
\hline Age at screening, years & 62.7 & 6.7 & 62.8 & 6.7 & .71 \\
\hline Physical functioning (RAND 36-Item Health Survey) & 82.1 & 19.0 & 80.9 & 18.9 & .18 \\
\hline \multicolumn{6}{|l|}{ Intervention related at baseline } \\
\hline Baseline weight, $\mathrm{kg}$ & 77.5 & 16.5 & 77.6 & 15.8 & .96 \\
\hline Dietary energy, kcal & $1,781.8$ & 694.4 & $1,769.5$ & 675.0 & .71 \\
\hline Energy from fat, \% & 37.6 & 4.9 & 37.8 & 5.0 & .38 \\
\hline Fruit and vegetable consumption, median servings/day & 3.8 & 1.8 & 3.6 & 1.8 & .08 \\
\hline Grains, median servings/day & 4.7 & 2.5 & 4.7 & 2.4 & .69 \\
\hline Change in grains consumption, median servings/day & 0.4 & 2.8 & -0.5 & 2.2 & $<.001$ \\
\hline \multicolumn{6}{|l|}{$\begin{array}{l}\text { Intervention related at last measure before breast cancer } \\
\text { diagnosis }\end{array}$} \\
\hline Time to diagnosis, years & 4.5 & 2.5 & 4.5 & 2.5 & .87 \\
\hline Age at breast cancer diagnosis, years & 67.5 & 6.9 & 67.6 & 6.9 & .66 \\
\hline Change in weight, $\mathrm{kg}$ & -1.2 & 10.0 & 0.5 & 8.5 & $<.001$ \\
\hline Change in energy intake, $\neq \mathrm{kcal} /$ day & -297.0 & 596.2 & -184.9 & 600.0 & $<.001$ \\
\hline Change in energy from fat,$\neq \% /$ day & -11.5 & 8.2 & -1.5 & 6.9 & $<.001$ \\
\hline $\begin{array}{l}\text { Change in fruit and vegetable consumption, } ¥ \text { median } \\
\text { servings/day }\end{array}$ & 1.4 & 2.2 & 0.2 & 1.7 & $<.001$ \\
\hline Change in grains consumption, median servings/day & -0.1 & 2.7 & -0.7 & 2.3 & $<.001$ \\
\hline
\end{tabular}

Abbreviations: MET, metabolic equivalent task; SD, standard deviation.

*Gail MH, Costantino JP, Pee D, et al: Projecting individualized absolute invasive breast cancer risk in African American women. J Natl Cancer Inst 99:1782-1792, 2007 tChange from baseline to year $1(n=1,591)$.

¥Change from baseline to last measure before breast cancer diagnosis $(n=1,530$ for change in dietary characteristics; $87 \%$ of breast cancers). 